\title{
ACCIONES DE ENFERMERÍA EN ATENCIÓN PRIMARIA ANTE LOS RIESGOS DE HIPERTENSIÓN DURANTE EL EMBARAZO
}

\section{ARTÍCULO DE REVISIÓN}

ADRIÃO, Iracely Santos ${ }^{1}$, BARBOSA, Marluce Sampaio Nobre ${ }^{2}$

ADRIÃO, Iracely Santos. BARBOSA, Marluce Sampaio Nobre. Acciones de enfermería en atención primaria ante los riesgos de hipertensión durante el embarazo. Revista Científica Multidisciplinar Núcleo do Conhecimento. Año 06, Ed. 09, Vol. 04, págs. 84-100. Septiembre 2021. ISSN: 2448-0959, Enlace de acceso: https://www.nucleodoconhecimento.com.br/salud/riesgos-de-hipertension, DOI: 10.32749/nucleodoconhecimento.com.br/salud/riesgos-de-hipertension

\section{RESUMEN}

La preeclampsia se presenta como uno de los síndromes hipertensivos que pueden afectar a las mujeres durante el embarazo, durante el parto y/o en el postparto hasta los 10 días, por lo que sigue siendo una patología que, al no diagnosticarse y tratarse adecuadamente, puede causar un gran daño e incluso provocar la muerte de la mujer embarazada y/o parturienta. El objetivo principal de este estudio es conocer la prevención de la preeclampsia en atención primaria por parte de la enfermería. Por lo tanto, la siguiente pregunta es: ¿cuáles son las acciones de enfermería en Atención Primaria frente a los riesgos de hipertensión durante el embarazo? El presente trabajo se basa en una investigación bibliográfica, con un enfoque cualitativo realizado a través del método de revisión integradora. Para recoger los datos, se realizó una investigación en las bases de datos: Google Scholar, Pubmed, Scielo y Medline. Los descriptores utilizados fueron: Riesgos. Gestación. Hipertensión. Enfermería. Los criterios de inclusión utilizados para la selección de muestras fueron: artículos disponibles publicados electrónicamente de 2015 a 2020; idioma portugués. Los

\footnotetext{
${ }^{1}$ Estudiante de Enfermería de la Universidad CEUMA.

2 Máster en Enfermedades Tropicales.
}

RC: 99507

Enlace de acceso: https://www.nucleodoconhecimento.com.br/salud/riesgos-de-hipertension 
criterios de exclusión fueron artículos inferiores a 2015 y palabras clave que no están relacionadas con el tema. Para el análisis completo de los artículos seleccionados, se utilizó una plataforma en línea (búsqueda en bases de datos), con el propósito de extraer (título y propósito de los artículos), organizar (en forma de tabla), resumir la información y facilitar la formación de la base de datos. Los resultados indicaron 35 artículos publicados, pero solo 15 artículos, en los últimos cinco años, tienen como tema principal la prevención y los riesgos de preeclampsia en atención primaria. Se concluye que las principales conductas utilizadas no solo por las enfermeras, sino por todo el equipo que trabaja en atención primaria, deben basarse en el proceso de acogida centrado en acciones que puedan ser determinantes en la prevención de la preeclampsia.

Palabras clave: Riesgos, Embarazo, Hipertensión, Enfermería.

\section{INTRODUCCIÓN}

Actualmente vivimos en un mundo profundamente marcado por la producción de conocimiento en todas las áreas, incluyendo la enfermería y la medicina. Incluso con esto, el número de mujeres afectadas por enfermedades ampliamente conocidas, como el Síndrome específico del embarazo hipertensivo (SHEG), sigue siendo muy grande. Su incidencia es alta, hasta el punto de ser tratada como un problema de salud pública (ARAÚJO, et al, 2017).

Si los síndromes hipertensivos relacionados con el estado de embarazo de las mujeres son tan importantes, por lo tanto, deben ser conocidos por las autoridades y los profesionales de la salud, y por lo tanto es de preguntarse, porque en el siglo 21, tales patologías todavía comprometen la salud y, a menudo, incluso la vida de las mujeres embarazadas y neonatos. Cuando se sabe que la práctica de exámenes prenatales identificaría el problema, o al menos permitiría su diagnóstico precoz, lo que en la práctica permitiría una intervención oportuna (ARNALDO; CARDOZO, 2021).

RC: 99507

Enlace de acceso: https://www.nucleodoconhecimento.com.br/salud/riesgos-de-hipertension 
Todas estas reflexiones contribuyeron y motivaron la elección del tema como objeto de estudio, ya que su viabilidad fue en parte el siguiente problema: ha habido una alta tasa de mujeres embarazadas que casi siempre son atendidas para presentar signos y síntomas de SHEG, especialmente preeclampsia. Entonces, ¿cuáles son las acciones de enfermería en Atención Primaria ante los riesgos de hipertensión durante el embarazo? Y como respuesta provisional al problema expuesto, se adoptó la hipótesis de que las mujeres en periodo gestacional e incluso parto y en el posparto, presentan alto índice de preeclampsia, debido a que no tuvieron un seguimiento prenatal adecuado (ARAÚJO et al., 2017).

Los estudios que involucran este conjunto de información (riesgos de hipertensión durante el embarazo), ayudan en la prevención de la hipertensión, ya que estimula y alerta a la población para que adopte cambios en los hábitos alimenticios y en la práctica habitual de actividad física, así como facilita el abordaje farmacológico de sus componentes aislados o síndrome metabólico en sí, a través de profesionales e investigadores (ARNALDO; CARDOZO, 2021).

La elección del tema actúa para acercar a las mujeres embarazadas información que las conduzca a una vida más saludable, pues esta es la principal tarea de los profesionales de la salud. Y para estas mujeres en embarazo deben comenzar a llevar a su día a día una dieta más sana y natural, teniendo como consejo el consumo de frutas, verduras, hortalizas. Estar orientado a minimizar el consumo de carne, grasas, frituras, sándwiches y otras formas de alimentos nocivos para la salud y que conduzcan a un aumento de la hipertensión (SOARES et al., 2015).

Para una buena calidad de vida, la mujer embarazada considerada hipertensa necesita mantener una vida saludable, debido a que muchos de estos se adhieren al tratamiento farmacológico, donde la presión arterial se controla a través de la ingestión de tabletas. También existe un tratamiento no farmacológico que se realiza a través de prácticas de actividad física, que también puede ser un complemento al tratamiento farmacológico (MELO et al., 2015). 
La práctica del ejercicio es de fundamental importancia para la salud y la calidad de vida en general y especialmente para las mujeres embarazadas, que tienen la costumbre de llevar una vida sedentaria, ya que se encuentra en el período de descanso de la profesión, y comienza el sedentarismo (SOARES et al., 2015).

El trabajo se justifica porque es de gran interés para la enfermería y la mujer que pretende ser madre. El interés en la investigación también fue motivado debido a que este es un tema que aporta información importante y fundamental a cada mujer que pretende quedar embarazada y para el área de actividad de enfermería.

Y así la investigación aporta enseñanzas a una buena calidad de vida de las mujeres embarazadas, ya que las mujeres hipertensas necesitan mantener una vida saludable, pues muchas de estas se adhieren al tratamiento farmacológico, donde la presión arterial se controla a través de la ingestión de comprimidos. También existe un tratamiento no farmacológico que se realiza a través de prácticas de actividad física, que también puede ser un complemento al tratamiento farmacológico (SANTOS; NETO, 2016).

El objetivo principal de este trabajo es conocer las conductas de prevención de la preeclampsia en atención primaria por parte de la enfermería.

\section{METODOLOGÍA}

En cuanto a los procedimientos metodológicos del presente estudio, se utilizó el desarrollo de la investigación bibliográfica, es decir, una revisión bibliográfica de artículos científicos sobre hipertensión y embarazo. Para la presente investigación científica, los artículos fueron buscados en las bases de datos de Google Scholar, Pubmed, Scielo y Medline, libros de revistas de salud y artículos científicos, basados en materiales ya elaborados.

Además, la investigación exploratoria para Lakatos y Marconi (2010, p. 171) "los estudios exploratorios-descriptivos combinados tienen como objetivos describir un fenómeno completamente cierto, como el estudio de un caso para el que se realizan análisis empíricos y teóricos".

RC: 99507

Enlace de acceso: https://www.nucleodoconhecimento.com.br/salud/riesgos-de-hipertension 
Por lo tanto, la investigación siempre busca la mejor aproximación del investigador con la situación real de los problemas, además, consiste en comprender de una manera menos totalitaria, fragmentando las opiniones presentadas y apuntando a la perspectiva de los actores que están involucrados en el proceso.

En relación con los medios, se aplicaron a este estudio los procedimientos de investigación bibliográfica. Así, las publicaciones nacionales, en libros, artículos de autoría de profesionales de enfermería y revistas formarán parte de la construcción de este material (FONSECA, 2002).

Por lo tanto, es notable que, a pesar de todo el esfuerzo por elevar las opiniones de los actores sociales involucrados en el proceso al que se propone estudiar la investigación, se percibe que la base teórica a través de la encuesta bibliográfica se convierte en la base para la comprensión de la investigación.

En este estudio, se planteó la comprensión de algunos autores a partir de la investigación bibliográfica en detalle en busca de la visión de este estudio. Enriquecer el conocimiento, porque es de fundamental importancia verificar la posición de estos autores respecto a la enfermería y los riesgos de hipertensión durante el embarazo.

Por lo tanto, los criterios de inclusión fueron: artículos publicados en portugués, que abordan la enfermería y los riesgos de hipertensión durante el embarazo, y que han sido publicados e indexados en estas bases de datos de 2015 a 2020. Los criterios de exclusión fueron artículos inferiores a 2015 y palabras clave que no están relacionadas con el tema. Las palabras clave utilizadas fueron: Riesgos. Gestación. Hipertensión. Enfermería. La recolección de datos también se realizó en la primera mitad de 2021.

La presente investigación tuvo entre sus fuentes de recolección de datos, a través de catalogación y carpeta de documentación bibliográfica, libros, artículos, revistas, periódicos, publicaciones periódicas, y artículos publicados en Internet, de autores contemporáneos, a través de los cuales se realizaron varias interpretaciones del tema. Por lo tanto, es importante mencionar, el medio de recopilación de datos relacionados, es lo que se espera cultivar de la mejor manera posible los orígenes y motivaciones de las respuestas encontradas o no al final de la investigación.

RC: 99507

Enlace de acceso: https://www.nucleodoconhecimento.com.br/salud/riesgos-de-hipertension 


\section{DESAROLLO}

La muestra final de esta revisión consistió en 15 (quince) artículos científicos, seleccionados de acuerdo con criterios de inclusión previamente establecidos. La Tabla 1 representa las especificaciones de cada uno de los artículos, distribuidas según: año; periódico; nombre y título de los autores.

Mesa uno. Lista de estudios seleccionados por año, revista, autores y título entre 2015 y 2020.

\begin{tabular}{|c|c|c|c|}
\hline ÑO & PERIÓDICO & AUTORES & TÍTULO \\
\hline 2017 & $\begin{array}{l}\text { Rev enferm UFPE } \\
\text { on line }\end{array}$ & $\begin{array}{l}\text { ARAÚJO, Isabella } \\
\text { Félix Meira, et al }\end{array}$ & $\begin{array}{l}\text { Síndromes hipertensivas e } \\
\text { fatores de risco associados à } \\
\text { gestação }\end{array}$ \\
\hline 21 & $\begin{array}{l}\text { Revista científica } \\
\text { multidisciplinar } \\
\text { núcleo do } \\
\text { conhecimento }\end{array}$ & $\begin{array}{ll}\text { ARNALDO, } & \text { Mariany } \\
\text { de } & \text { Freitas. } \\
\text { CARDOZO, } & \\
\text { Maryanne Neuraide } \\
\text { Freire }\end{array}$ & $\begin{array}{l}\text { Assistência de enfermagem } \\
\text { às gestantes hipertensas na } \\
\text { prevenção da prematuridade: } \\
\text { revisão bibliográfica }\end{array}$ \\
\hline 19 & $\begin{array}{ll}\text { Trabalho } & \text { de } \\
\text { Conclusão } & \text { de } \\
\text { Curso } & \end{array}$ & $\begin{array}{l}\text { CABRAL, Gustavo } \\
\text { Pereira }\end{array}$ & $\begin{array}{l}\text { Cuidados de enfermagem a } \\
\text { mulheres com doença } \\
\text { hipertensiva específica da } \\
\text { gestação: uma revisão } \\
\text { integrativa }\end{array}$ \\
\hline 017 & $\begin{array}{l}\text { International } \\
\text { Nursing Congress }\end{array}$ & $\begin{array}{ll}\text { ENDRINGER, Deyvid } \\
\text { Dantas; } \quad \text { CRUZ, } \\
\text { Monielle Lima }\end{array}$ & $\begin{array}{l}\text { Representatividade do } \\
\text { enfermeiro na assistência a } \\
\text { gestantes com pré-eclâmpsia }\end{array}$ \\
\hline 2015 & $\begin{array}{lr}\text { REBES - } & \text { Revista } \\
\text { Brasileira } & \text { de } \\
\text { Educação e Saúde }\end{array}$ & $\begin{array}{l}\text { MELO, Wyara } \\
\text { Ferreira, et al }\end{array}$ & $\begin{array}{l}\text { A hipertensão gestacional e o } \\
\text { risco de pré-eclampsia: } \\
\text { revisão bibliográfica }\end{array}$ \\
\hline 018 & $\begin{array}{l}\text { Brazilian Journal of } \\
\text { Surgery and Clinical }\end{array}$ & $\begin{array}{l}\text { OLIVEIRA, Leilyanne } \\
\text { de Araújo Mendes, et }\end{array}$ & $\begin{array}{l}\text { Cuidados de enfermagem a } \\
\text { gestante com síndrome }\end{array}$ \\
\hline
\end{tabular}




\begin{tabular}{|c|c|c|c|}
\hline & Research - BJSCR & al & $\begin{array}{l}\text { hipertensiva: } \quad \text { revisão } \\
\text { integrativa. }\end{array}$ \\
\hline 2018 & $\begin{array}{l}\text { Revista Científica } \\
\text { Multidisciplinar } \\
\text { Núcleo do } \\
\text { Conhecimento }\end{array}$ & $\begin{array}{l}\text { SANTOS, Keilane } \\
\text { Carvalho. } \\
\text { BRASILEIRO, } \\
\text { Marislei Espíndula }\end{array}$ & $\begin{array}{l}\text { Enfermagem e os cuidados } \\
\text { Emergenciais na doença } \\
\text { hipertensiva específica na } \\
\text { gravidez }\end{array}$ \\
\hline 2016 & $\begin{array}{l}\text { Revista Científica } \\
\text { da FASETE }\end{array}$ & $\begin{array}{l}\text { SANTOS, Joelma } \\
\text { Oliveira Silva; NETO, } \\
\text { Thiago Paulo de } \\
\text { Almeida }\end{array}$ & $\begin{array}{l}\text { Atuação do enfermeiro na } \\
\text { redução da ocorrência da pré- } \\
\text { eclâmpsia: uma revisão } \\
\text { integrativa }\end{array}$ \\
\hline 2020 & Enfermagem Brasil & $\begin{array}{l}\text { SARMENTO, Rayani } \\
\text { Silva, et al }\end{array}$ & $\begin{array}{l}\text { Pré-eclâmpsia na gestação: } \\
\text { ênfase na assistência de } \\
\text { enfermagem }\end{array}$ \\
\hline 2016 & $\begin{array}{l}\text { Centro Universitário } \\
\text { Ritter dos Reis }\end{array}$ & $\begin{array}{lr}\text { TEIXEIRA, Rafaela da } \\
\text { Rosa; } & \text { MINUZZI, } \\
\text { Renata } & \text { Nolibus; } \\
\text { TELES, } & \text { Jéssica } \\
\text { Machado } & \end{array}$ & $\begin{array}{l}\text { Cuidados de enfermagem à } \\
\text { pacientes com hipertensão } \\
\text { gestacional: um relato de } \\
\text { experiência }\end{array}$ \\
\hline 2017 & $\begin{array}{l}\text { Rev Port Med Geral } \\
\text { Fam }\end{array}$ & $\begin{array}{l}\text { FERREIRA, Sara } \\
\text { Santos, et al }\end{array}$ & $\begin{array}{l}\text { Ácido acetilsalicílico na } \\
\text { prevenção da pré-eclâmpsia: } \\
\text { uma revisão baseada na } \\
\text { evidência }\end{array}$ \\
\hline 2015 & $\begin{array}{l}\text { Ciência } \\
\text { Enfermeria }\end{array}$ & $\begin{array}{l}\text { KRAUZER, Ivete } \\
\text { Maroso, et al. }\end{array}$ & $\begin{array}{l}\text { Sistematização da } \\
\text { assistência de enfermagem } \\
\text { na atenção básica: o que } \\
\text { dizem os enfermeiros? }\end{array}$ \\
\hline 2015 & $\begin{array}{l}\text { J. res.: fundam. } \\
\text { care. }\end{array}$ & $\begin{array}{l}\text { FERNANDES, Amélia } \\
\text { Carolina Lopes }\end{array}$ & $\begin{array}{l}\text { Sistematização da } \\
\text { assistência de enfermagem } \\
\text { na prevenção de infecções } \\
\text { em unidade de terapia } \\
\text { intensiva }\end{array}$ \\
\hline
\end{tabular}




\begin{tabular}{|l|l|l|l|}
\hline 2015 & $\begin{array}{l}\text { Escola Anna Nery } \\
\text { Revista } \\
\text { Enfermagem }\end{array}$ & $\begin{array}{l}\text { SOARES, Mirelle } \\
\text { Inácio, et al }\end{array}$ & $\begin{array}{l}\text { Sistematização da } \\
\text { assistência de enfermagem: } \\
\text { facilidades e desafios do } \\
\text { enfermeiro na gerência da } \\
\text { assistência }\end{array}$ \\
\hline 2016 & Rev. Interd & $\begin{array}{l}\text { MONTEIRO, M. M., et } \\
\text { al. }\end{array}$ & $\begin{array}{l}\text { Emergências obstétricas: } \\
\text { características de casos } \\
\text { atendidos por serviço móvel } \\
\text { de urgência }\end{array}$ \\
\hline
\end{tabular}

Fuente: Autoría propia (2021)

\subsection{CARACTERÍSTICAS DE LA HIPERTENSIÓN EN EL EMBARAZO}

En esta investigación tendremos énfasis en una patología que es la segunda causa principal de muerte perinatal en Brasil, que es la hipertensión en el embarazo, en la que conlleva varios riesgos para la madre y el niño, por lo que es necesario seguir regularmente a estos pacientes y guiar los cambios en el estilo de vida y la dieta (ARAÚJO et al., 2017).

La presión arterial (PA) presenta alteraciones en la interacción de factores neurohumorales, conductuales y ambientales. La hipertensión (AH) puede conceptualizarse como un síndrome de origen multifactorial, considerado como uno de los principales problemas en el área de la salud pública siendo evaluado como un factor de riesgo grave para las enfermedades cardiovasculares (ARNALDO; CARDOZO, 2021).

Las mujeres que usan cigarrillos y anticonceptivos, mayores de 30 años, terminan siendo las más afectadas, porque son sumisas a otras enfermedades pulmonares. Las mujeres reciben protección debido a eventos cardiovasculares que ocurren antes de la menopausia, en los hombres solo surgen después de los 30 años (SOARES et al., 2015). 
Los estudios a más largo plazo han identificado que no son diferentes de los hombres con respecto a la respuesta antihipertensiva de la presión arterial; sin embargo, es de suma importancia evitar el uso de inhibidores enzimáticos de angiotensina para usar (ECA) y antagonistas de los receptores de angiotensina II en mujeres cuando están en edad fértil y que no están utilizando métodos anticonceptivos seguros comprobados (SANTOS; NETO, 2016).

Las causas de la hipertensión se asocian con una vida sedentaria, con una ingesta inadecuada de alimentos, como el consumo excesivo de sodio y grasa o alcohol, e incluso el tabaquismo, que es la causa de la insuficiencia cardíaca. El hecho es que la hipertensión es un potente contribuyente a las enfermedades cardiovasculares, atacando y sobrecargando el corazón hasta que un día falla (CABRAL, 2019).

La hipertensión en el embarazo es la segunda causa de muerte materna y perinatal en Brasil según datos del Ministerio de Salud. Esto demuestra la necesidad de conocer y controlar esta patología. En el embarazo el organismo de la mujer sufre varias transformaciones para poder desarrollar el feto que estará resguardado durante unos 280 días o aproximadamente 9 meses. En Brasil, se espera que la población alcance la marca de 228 millones de habitantes en 2025 (CABRAL, 2019).

La tasa de mortalidad según el IBGE en 2016 es de 6,6 nacidos vivos por cada mil habitantes. Ante estos datos, es evidente la necesidad de desarrollar investigación y diseño para mejorar este alarmante índice, a partir del mapeo de los lugares que tienen mayor riesgo gestacional, lo que puede intensificar la atención a las gestantes y así mejorar la tasa de mortalidad (ENDRINGER; CRUZ, 2017).

\subsection{ASPECTOS GENERALES DE LA PRE-ECLÂMPSIA}

No se sabe a ciencia cierta cuándo se identificaron los primeros casos de esta patología, pero lo que sí se sabe es que se ha descrito desde los tiempos en que se comenzaron a hacer registros, su ocurrencia se atribuyó a malos espíritus, mal genio $\mathrm{y}$, más recientemente, ingesta de sodio y aumento de peso durante el embarazo (ENDRINGER; CRUZ, 2017).

RC: 99507

Enlace de acceso: https://www.nucleodoconhecimento.com.br/salud/riesgos-de-hipertension 
Se observa que este síndrome ha causado trastornos importantes en mujeres jóvenes embarazadas correlacionados con el primer embarazo, y se caracteriza como la principal causa de mortalidad materna en Brasil. En 1998, la toxemia del embarazo fue responsable del $21 \%$ de las muertes obstétricas directas (SANTOS; BRASILEIRO, 2018).

La preeclampsia se caracteriza por ser uno de los síndromes hipertensivos que pueden afectar a las mujeres durante el embarazo, durante el parto y/o en el puerpo hasta los 10 días, por lo que sigue siendo una patología que, al no diagnosticarse y tratarse adecuadamente, puede causar grandes daños e incluso provocar la muerte de la mujer embarazada y/o parturienta (SARMENTO et al., 2020).

Se caracteriza como una enfermedad sistémica que surge a partir de la semana $20^{\mathrm{a}}$ de gestación por la tríada: hipertensión, edema y proteinuria y puede tener su condición agravada por eclampsia, en presencia de convulsión, y/o evolucionar al síndrome HELLP (SANTOS; BRASILEÑO, 2018).

Las causas de la preeclampsia aún se desconocen, sin embargo, lo que sí se sabe es que algunas mujeres embarazadas tienen más probabilidades de verse afectadas por esta patología. En la antigüedad se referían a los malos genios, el aumento de peso y el consumo excesivo de sal (FERREIRA et al., 2017).

Hoy en día aún no se conoce a ciencia cierta los motivos causales y desencadenantes de la preeclampsia, lo que sí se sabe es que tiene multicausales: predisposición familiar y genética, endocrino-metabólica, incluyendo alteración de la producción de prostaglandinas, isquemia uteroplacental, inmunológica, presencia de tejido trofoblástico, toxinas en el torrente sanguíneo materno, causando vasoconstricción y placenta defectuosa (SARMENTO et al., 2020).

La preeclampsia tiene varias etiologías que pueden provenir de causas hereditarias y/o adquiridas debido al estilo de vida del paciente o a las condiciones sociales y ambientales. Sin embargo, lo que sí se sabe es que las condiciones sociales, junto con una vida sedentaria, una mala alimentación, y la falta de información 
generalmente derivada de la baja escolaridad, favorecen el riesgo inminente de aparición de preeclampsia, además de otras (TEIXEIRA; MINUZZI; TELES, 2016).

El síndrome hipertensivo se presenta inicialmente como preeclampsia leve, luego progresa a preeclampsia severa, eclampsia, síndrome de Hellp, CIVD (coagulación intravascular diseminada) y finalmente la muerte. $Y$ estas son las etapas de gravedad del síndrome hipertensivo, sin embargo, no necesariamente necesita pasar por todas las etapas, y puede evolucionar de una preeclampsia leve al síndrome de Hellp (FERREIRA et al., 2017).

Otras complicaciones de la preeclampsia son: desprendimiento prematuro de placenta como resultado de una lesión placentaria en el sitio de implantación, edema pulmonar agudo, insuficiencia renal aguda por glomerulopatía instalada y hemorragia cerebral (TEIXEIRA; MINUZZI; TELES, 2016).

Los radicales libres de $\mathrm{O} 2$ se identifican con células grasas que son endotelio, solubles en sangre materna y muy cercanas al endotelio vascular. Reducen la producción de prostaciclina y óxido nítrico que son vasos dilatados, y aumentan la resistencia vascular, causando un aumento de la presión arterial (FERREIRA et al., 2017).

La única alternativa para controlar la preeclampsia y prevenir la eclampsia es el correcto seguimiento de la atención prenatal durante el embarazo. Los pacientes con preeclampsia leve deben optar por el reposo, medir siempre la frecuencia de la presión arterial y seguir una dieta baja en sal (KRAUZ et al., 2015).

Los fármacos antihipertensivos y anticonvulsivos están indicados para el control de la eclampsia con mayor gravedad, que puede requerir la anticipación del parto. Y la enfermedad tiende a retroceder espontáneamente con la extirpación de la placenta (FERNANDES, 2015).

Por lo tanto, según Varella (2020, p. 1), es importante seguir algunas recomendaciones:

- Se dirigir ao ginecologista antes de engravidar para uma avaliação clínica e início da administração de ácido fólico;

RC: 99507

Enlace de acceso: https://www.nucleodoconhecimento.com.br/salud/riesgos-de-hipertension 
- Importante estar presente em todas as consultas previstas no pré-natal;

- Qualquer desatenção e a ausência de sintomas podem fazer com que uma forma leve de pré-eclâmpsia evolua com complicações;

- Realizar exercícios físicos de acordo com a fase da gestação;

- Reduzir a quantidade de sal nas refeições, não fumar e principalmente não ingerir álcool durante a gravidez (VARELLA, 2020, p. 1).

El tratamiento consiste en reposo en cama (lado izquierdo), en un lugar aireado y tranquilo, con el uso de medicamentos, con la intención de minimizar el empeoramiento y posponer el parto prematuro, cuando sea posible, es decir, cuando no haya un riesgo inminente para la madre y el feto. Sin embargo, cuando esto no es posible, la indicación es la interrupción inmediata del embarazo, independientemente del período gestacional (FERREIRA et al., 2017).

\subsection{CUIDADOS DE ENFERMERÍA EN LOS NIVELES DE ATENCIÓN DE LA SALUD DE LA MUJER}

La Sistematización de los Cuidados de Enfermería (SAE) apunta a un cuidado de manera amplia, dinámica, con una planificación que involucre los procesos de enfermería en su conjunto, cuyo cuidado cuenta con la participación armónica de varios profesionales junto con la familia y el paciente, construyendo un ambiente propicio para la recuperación exitosa y con menos desgaste tanto para el pacientefamilia como para el equipo (SOARES et al., 2015).

El cuidado de enfermería también apunta a la integración en la sociedad, donde su enfoque principal es proporcionar calidad de vida a las personas, con el fin de conducir a un mejor y más amplio cuidado de sus necesidades específicas, priorizando a las personas que están bajo cuidados y atención de enfermería, es decir, aquellos que ya están haciendo un seguimiento (MONTEIRO et al., 2015).

"Enfermería Planificada" busca llevar a cabo una orientación profesional respecto a la elaboración de una planificación sistemática de los cuidados de enfermería. La serie de principios aquí coordinados se atribuye a la nomenclatura del "Plan de Enfermería

RC: 99507

Enlace de acceso: https://www.nucleodoconhecimento.com.br/salud/riesgos-de-hipertension 
Terapéutica", un plan que compone todo el proceso de acciones y decisiones dirigidas a programar los cuidados de enfermería. (SOARES et al., 2015, p. 50).

Se entiende que el cuidado de enfermería es todo cuidado o técnica dispensada a un paciente, y cada procedimiento que reduce o minimiza el dolor. Cuando la enfermera cuida a un paciente, dispensa no solo atención, sino que se conecta con el cliente para reducir su dolor, proporcionando así su recuperación, reduciendo sus lesiones y fomentando su mejoría tanto física como mental, a través de la acción junto con el equipo de salud y la familia (MONTEIRO, et al., 2015).

Sin embargo, la planificación de los cuidados de enfermería es una misión que presenta un cierto grado de complejidad debido a varios factores: uno de estos factores es que el proceso de planificación en enfermería implica la participación armónica de varias personas, como el paciente, la enfermera y su equipo y los miembros del equipo de salud; otra razón es la indispensabilidad del uso del razonamiento, la toma de decisiones, el rendimiento y la documentación; además de estos, otra razón preponderante está relacionada con los cambios constantes en la vida que afectan incluso a los planes más mejorados (SOARES et al., 2015).

Se observa que para realizar un buen cuidado de enfermería es necesario tener en cuenta varios factores, siendo uno de los principales factores la capacidad de las enfermeras para realizar trabajos en conjunto con el equipo de salud, cuidando paradigmas del paciente y su familia. Utilizar su capacidad para transmitir información y, por otro lado, saber escuchar, y esto constituye un componente básico para un buen profesional de enfermería (MONTEIRO et al., 2015).

La enfermera de atención forma parte de un equipo multidisciplinario que apunta a acciones preventivas y curativas dentro de la comunidad a través de la promoción de políticas de salud orientadas a reducir las notificaciones de daños, a través del conocimiento de las causas y riesgos a los que está expuesta la población, y al mismo tiempo, sensibilizar a los organismos públicos sobre las acciones que deben implementarse (KRAUZER, 2015). 
Al realizar la anamnesis en un cliente determinado, se debe adoptar una mirada reflexiva y holística dirigida no solo a la patología subyacente o a la que está en evidencia, sino también a tratar de comprender las razones por las que surgió, con el fin de buscar una solución para el paciente (SOARES et al., 2015).

En esta recolección de datos, las enfermeras deben elaborar planes de atención sistematizados con el fin de promover y recuperar la salud, que había sido sacudida, apuntando a un diagnóstico correcto. Para Carpenito apud Fernandes, et al (2015, p. 1.581):

O diagnóstico de enfermagem baseia-se tanto nos problemas reais (voltados para o presente) quanto nos problemas potenciais (voltados para o futuro), que podem ser sintomas de disfunções fisiológicas, comportamentais, psicossociais ou espirituais.

Se entiende que el diagnóstico de enfermería se divide en dos momentos: un diagnóstico real que se caracteriza por las manifestaciones definidas ya existentes en el individuo, es decir, movilidad física deteriorada, relacionada con la lesión de las extremidades inferiores. El segundo es que el diagnóstico de riesgo se caracteriza por factores de riesgo a los que está expuesto el individuo, vulnerable tanto por el entorno como por su propia inmunidad física, es decir, por el riesgo de infección relacionado con el tiempo de hospitalización (KRAUZER, 2015).

\subsection{CUIDADOS DE ENFERMERÍA Y ACCIONES EN LA PREVENCIÓN DE LA PREECLAMPSIA}

Durante la atención prenatal se solicitan algunas pruebas y a través de estas se pueden detectar las patologías agudas o crónicas que se dirigen al tratamiento para no perjudicar el ciclo gestacional y disminuir las tasas de morbilidad y mortalidad materno-infantil (ARNALDO; CARDOZO, 2021).

La consulta prenatal debe llevarse a cabo al principio del embarazo, ya que se puede realizar una evaluación más detallada de las condiciones maternas y fetales. Incluso si el embarazo se considera un proceso fisiológico normal, se producen varios tipos de cambios en el cuerpo de la mujer y esto causa una distancia más corta entre la RC: 99507

Enlace de acceso: https://www.nucleodoconhecimento.com.br/salud/riesgos-de-hipertension 
salud y la enfermedad que cuando no está embarazada. La prevención o al menos el diagnóstico precoz de signos anormales, e inmediatamente un tratamiento eficaz, puede actuar como una estrategia para minimizar diversos tipos de complicaciones asociadas con el parto, no solo durante el período antes del parto, sino también durante el parto (ENDRINGER; CRUZ, 2017).

Se sabe que el profesional de la salud debe estar al tanto de cualquier signo que pueda indicar una situación que comprometa la atención prenatal, por esta razón la enfermera debe realizar todos los pasos, además de la solicitud de pruebas de laboratorio (SANTOS; BRASILEIRO, 2018).

La enfermería siempre debe prestar atención durante todo el período gestacional para que cualquier ocurrencia no planificada no se manifieste durante este evento. Las acciones de promoción de la salud y prevención de riesgos durante el embarazo deben centrarse en atender a esta clientela mucho más específica que requiere todos los cuidados. En este contexto, la atención prenatal, tal como lo recomienda el Ministerio de Salud, sirve como la principal forma de generar la seguridad que los clientes y profesionales de la salud necesitan tener sobre la consolidación de un embarazo libre de riesgos y complicaciones (MONTEIRO et al., 2016).

En este sesgo, la consulta de enfermería, el seguimiento de la gestación durante la atención prenatal, la atención a cualquier manifestación clínica fuera de lo planificado $y$ el asesoramiento funcionan como fundamentales y necesitan recibir un seguimiento adecuado. Por otro lado, Ilaman la atención sobre las complicaciones relacionadas con la hipertensión arterial, la presencia de hemorragias, hemorragias y signos de preeclampsia y eclampsia, como faros de complicaciones más profundas y mortalidad (SANTOS; BRASILEIRO, 2018).

La preeclampsia tiene su inicio lento y gradual con complicaciones importantes para la madre y el feto, por lo que es necesario que exista una atención prenatal de buena calidad que brinde acciones preventivas y la identificación de casos predisponentes con el fin de diagnosticar lo antes posible, para iniciar el tratamiento profiláctico. Los 
eventos también deben registrarse en los registros médicos para que esta información no se pierda y facilite la atención futura (MONTEIRO et al., 2016).

La enfermera profesional debe realizar ya en la primera consulta con la mujer embarazada, una anamnesis completa obteniendo la mayor cantidad de información relevante para su salud actual y anterior, además de antecedentes familiares, y para ello debe solicitar pruebas de rutina, apoyo vitamínico, orientación sobre su dieta, llenar su tarjeta de embarazada, revisar su tarjeta de vacunación y calendario para la próxima consulta (ENDRINGER; CRUZ, 2017).

El cuidado de enfermería en el período prenatal es de suma importancia porque monitorea e identifica posibles cambios en su período inicial, y remite a una evaluación más precisa en una unidad de referencia, con esta correcta toma de decisiones puede marcar la diferencia, y brindar a la gestada mayor seguridad y caracterizar un verdadero cuidado preventivo, vale la pena mencionar que el profesional de enfermería es un protagonista de incalculable importancia en la atención prenatal, porque es precisamente porque tiene el mayor vínculo con las mujeres embarazadas (SANTOS; BRASILEIRO, 2018).

\section{CONSIDERACIONES FINALES}

El estudio, construido a partir de una revisión de literatura especializada, permitió un análisis sobre la prevención de la preeclampsia en atención primaria, y para ello, realizó una breve historia sobre la incidencia de esta patología, así como sus conceptos y características.

Además, fue apropiado describir las contribuciones de los cuidados de enfermería en la Atención Primaria. Por lo tanto, se entiende que para realizar un buen cuidado de enfermería es necesario tener en cuenta varios factores, siendo uno de los principales factores la capacidad de las enfermeras para realizar trabajos en conjunto con el equipo de salud, cuidando paradigmas del paciente y su familia. Utilizar su capacidad para transmitir información y por otro lado saber escuchar, y esto constituye componentes básicos para un buen profesional de enfermería. 
La preeclampsia, aunque es una patología o un síndrome que puede afectar a las mujeres en estado de embarazo, todavía se caracteriza como un problema de salud pública, a pesar de todo el desarrollo de la ciencia, incluso en el área de enfermería y en el área médica, precisamente la razón desencadenante del estudio, que parte del problema propuesto en la introducción, que, según los investigadores, fue tratado en profundidad, de manera que los datos obtenidos fueron consistentes y los resultados de los mismos pueden generalizarse a toda la población estudiada.

Así, utilizando artículos que se encontraron centrados en el tema de este estudio, surge la necesidad de realizar muchas otras publicaciones sobre la prevención de la preeclampsia. La mayoría de los estudios se centran más en el papel de las enfermeras en la humanización del contacto profesional/usuario en el momento del dolor agudo o crónico en los servicios de urgencias que en la prevención de la preeclampsia en la atención primaria.

Entonces, ¿cuáles son las acciones de enfermería en Atención Primaria ante los riesgos de hipertensión durante el embarazo? En respuesta al problema, vale la pena señalar que el profesional de enfermería que trabaja en una unidad de salud debe realizar conferencias con el fin de crear conciencia sobre la importancia de la atención prenatal lo más rápido posible para garantizar un embarazo saludable y una buena evolución del concepto y el parto.

En este sentido, se concluye que las principales conductas utilizadas no solo por las enfermeras, sino por todo el equipo que trabaja en atención primaria, deben estar centradas en el proceso de recepción, centradas en acciones que puedan ser determinantes en la prevención de la preeclampsia. Por lo tanto, es extremadamente importante publicar más estudios centrados en la prevención de la preeclampsia en la atención primaria.

RC: 99507

Enlace de acceso: https://www.nucleodoconhecimento.com.br/salud/riesgos-de-hipertension 


\section{REFERENCIAS}

ARAÚJO, Isabella Félix Meira, et al. Síndromes hipertensivas e fatores de risco associados à gestação. Rev enferm UFPE on line., Recife, 11(Supl. 10):4254-62, out., 2017.

ARNALDO, Mariany de Freitas. CARDOZO, Maryanne Neuraide Freire. Assistência de enfermagem às gestantes hipertensas na prevenção da prematuridade: revisão bibliográfica. Revista científica multidisciplinar núcleo do conhecimento. Ano 06, Ed. 02, Vol. 02, pp. 108 - 125 . Fevereiro de 2021.

CABRAL, Gustavo Pereira. Cuidados de enfermagem a mulheres com doença hipertensiva específica da gestação: uma revisão integrativa. Trabalho de Conclusão de Curso. Uruguaiana, 2019.

ENDRINGER, Deyvid Dantas; CRUZ, Monielle Lima. Representatividade do enfermeiro na assistência a gestantes com pré-eclâmpsia. International Nursing Congress. May 9-12, 2017.

FERNANDES, Amélia Carolina Lopes. Sistematização da assistência de enfermagem na prevenção de infecções em unidade de terapia intensiva. J. res.: fundam. care. online 2015. out./dez. 6(4):1580-1589.

FERREIRA, Sara Santos, et al. Ácido acetilsalicílico na prevenção da pré-eclâmpsia: uma revisão baseada na evidência. Rev Port Med Geral Fam 2017;33:118-32.

FONSECA, J. J. S. Metodologia da pesquisa científica. Fortaleza: UEC, 2002.

GIL, Antônio Carlos. Como elaborar projetos de pesquisa. 4. ed. São Paulo: Atlas, 2007.

KRAUZER, Ivete Maroso, et al. Sistematização da assistência de enfermagem na atenção básica: o que dizem os enfermeiros? Ciência Y Enfermeria XXI (2), 2015. 
LAKATOS, Eva Maria; MARCONI, Marina de Andrade. Fundamentos da metodologia científica. 7. ed. São Paulo: Atlas, 2010.

MELO, Wyara Ferreira, et al. A hipertensão gestacional e o risco de pré-eclampsia: revisão bibliográfica. REBES - Revista Brasileira de Educação e Saúde (Pombal - PB, Brasil), v. 5, n. 3, p. 07-11, jul-set, 2015.

MONTEIRO, M. M., et al. Emergências obstétricas: características de casos atendidos por serviço móvel de urgência. Rev. Interd. v. 9, n. 2, p. 136-144, abr/mai/jun. 2016 143.

OLIVEIRA, Leilyanne de Araújo Mendes, et al. Cuidados de enfermagem a gestante com síndrome hipertensiva: revisão integrativa. Brazilian Journal of Surgery and Clinical Research - BJSCR. Vol.23,n.2,pp.159-164 (Jun - Ago 2018).

SANTOS, Keilane Carvalho. BRASILEIRO, Marislei Espíndula. Enfermagem e os cuidados Emergenciais na doença hipertensiva específica na gravidez. Revista Científica Multidisciplinar Núcleo do Conhecimento. Ano 03, Ed. 11, Vol. 08, pp. 17-26 Novembro de 2018.

SANTOS, Joelma Oliveira Silva; NETO, Thiago Paulo de Almeida. Atuação do enfermeiro na redução da ocorrência da pré-eclâmpsia: uma revisão integrativa. Revista Científica da FASETE 2016.1.

SARMENTO, Rayani Silva, et al. Pré-eclâmpsia na gestação: ênfase na assistência de enfermagem. Enfermagem Brasil 2020;19(3):261-267.

SILVA, E.L. da; MENEZES, E.M. Metodologia da pesquisa e elaboração de dissertação. Florianópolis: Laboratório de Ensino à Distância da UFSC, 2001.

SOARES, Mirelle Inácio, et al. Sistematização da assistência de enfermagem: facilidades e desafios do enfermeiro na gerência da assistência. Escola Anna Nery Revista de Enfermagem 19(1) Jan-Mar 2015. 
TEIXEIRA, Rafaela da Rosa; MINUZZI, Renata Nolibus; TELES, Jéssica Machado. Cuidados de enfermagem à pacientes com hipertensão gestacional: um relato de experiência. XII Semana de Extensão, Pesquisa e Pós-Graduação SEPesq - 24 a 28 de outubro de 2016

Publicado: Agosto de 2021.

Aprobado: Septiembre de 2021. 